\title{
Ad hoc wireless sensor networks for exploration of Solar-system bodies
}

\author{
Philippe Dubois $^{\mathrm{a}, *}$, Cyril Botteron ${ }^{\mathrm{b}, 1}$, Valentin Mitev ${ }^{\mathrm{c}, 2}$, Carlo Menon ${ }^{\mathrm{d}, 3}$, \\ Pierre-André Farine $^{\mathrm{b}, 4}$, Paolo Dainesi ${ }^{\mathrm{e}, 5}$, Adrian Ionescu ${ }^{\mathrm{e}, 6}$, Herbert Shea ${ }^{\mathrm{a}, 7}$ \\ ${ }^{a}$ Microsystems for Space Technologies Laboratory, Ecole Polytechnique Fédérale de Lausanne, Lausanne, Switzerland \\ ${ }^{\mathrm{b}}$ Institute of Microtechnology (IMT), University of Neuchâtel, Switzerland \\ ${ }^{\mathrm{c}}$ Observatory of Neuchâtel, Neuchâtel, Switzerland \\ ${ }^{\mathrm{d}}$ School of Engineering Science, Simon Fraser University, Canada \\ e LEG2, Ecole Polytechnique Fédérale de Lausanne, Lausanne, Switzerland
}

Received 19 October 2007; accepted 3 November 2008

Available online 31 December 2008

\begin{abstract}
In this work, we evaluate the exploration of the Solar system by ad hoc wireless sensor networks (WSN), i.e., networks where all nodes (either moving or stationary) can both provide and relay data. The two aspects of self-organization and localization are the major challenges to achieve a reliable network for a variety of missions. We point out the diversity of environmental and operational constrains that WSN used for space exploration would face.

We evaluate two groups of scenarios consisting in static or moving sensing nodes that can be either located on the ground or in the atmosphere of a Solar-system object. These scenarios enable collecting data simultaneously over a large surface or volume.

We consider physical and chemical sensing of the atmosphere, surface and soil using such networks. Emerging technologies such as nodes localization techniques are reviewed. Finally, we compare the specific requirements of WSN for space exploration with those of WSN designed for terrestrial applications.
\end{abstract}

(C) 2008 Elsevier Ltd. All rights reserved.

Keywords: Wireless sensor network; WSN; Localization; Space and Solar-system exploration; Motes

\footnotetext{
* Corresponding author. Tel.: +41327205266; fax: +41327205754

E-mail addresses: philippe.dubois@unine.ch (P. Dubois), cyril.botteron@unine.ch (C. Botteron), Valentin.Mitev@csem.ch (V. Mitev), cmenon@sfu.ca (C. Menon), pierre-andre.farine@unine.ch (P.-A. Farine),paulo.dainesi@epfl.ch (P. Dainesi), Adrian.Ionescu@epfl.ch (A. Ionescu), herbert.shea@unine.ch (H. Shea)

${ }^{1}$ Tel.: 41327183424 ; fax: +41327183402

2 Tel.: 41328898813 ; fax: +41327220420

${ }^{3}$ Tel.: 17787826860 ; fax: +17787824951

${ }^{4}$ Tel.: 41327183404 ; fax: +41327183402

${ }^{5}$ Tel.: 41216935654 ; fax: +41216933640

${ }^{6}$ Tel.: 41216933978 ; fax: +41216933640

7 Tel.: 41327205584 ; fax: +41327205754
}

\section{Introduction}

One can distinguish two main applications of wireless sensor networks (WSN) in space: (1) implementation of WSN within a spacecraft in order to replace electrical wires and to enhance the robustness and functionalities of the mission or (2) direct scientific measurements with distributed WSN on, in, or around Solar-system bodies [1].

In the former case, wireless networks (WN) will increasingly be used within satellites, spacecraft and launchers in order to reduce the number and mass of cables for the data bus. In some cases, measuring 
certain physical quantities and transmitting them to a central processing unit could be useful. Simple WSN in launchers or spacecraft could be used to detect impending failures of the structures or components, for instance by detecting changes in the resonance frequencies. However, the approach generally followed in satellites is oriented toward a wireless data bus that transmits all the data from the various subsystems to a central processor. These data could come from complex sensing elements, i.e., high resolution imagers, or from microprocessors, and the data flow can therefore be much higher than the one produced by the simple sensors of a WSN.

In the latter case, distributed smart monitoring with WSN for the exploration of the Solar system has started to gain interest in the view of ESA [1]. Compared to single instruments, WSN for exploration are very promising in terms of cost reduction, reduced set-up time of a mission, redundancy, and scientific interest of the data. However, to date no mission based on WSN for exploration has been flown. WSN based exploration mission can address either atmospheric or ground based measurements. Such missions would require robust nodes capable of acquiring valid data in harsh environments while communicating with other nodes over large distances, i.e., a few kilometers. Depending on the mission, the requirements can be very different, but in general self-organization and localization of the WSN are needed. In contrast to single probe based mission, the success of a WSN based mission relies on the robustness of the WSN which is challenging to fully test on Earth. However, some Earth based tests can approach the situations that would be encountered in space by the WSN.

\section{Expected benefits from the introduction of WSN in space}

\subsection{Scientific benefits}

The scientific and technological differences, advantages and drawbacks of WSN for space exploration are best shown when comparing those missions to single probe/instrument based missions. Fig. 1 shows that exploration of space with WSN would not necessarily tend to replace single probe missions, but could provide different types of data that would enable to map accurately an area or volume of interest with simple sensors over a long period of time if necessary. WSN are thus likely to provide new data that would be difficult to collect with other methods based on single probe (Table 1).

Large size single probes can contain essentially three types of instruments: complex local sensor

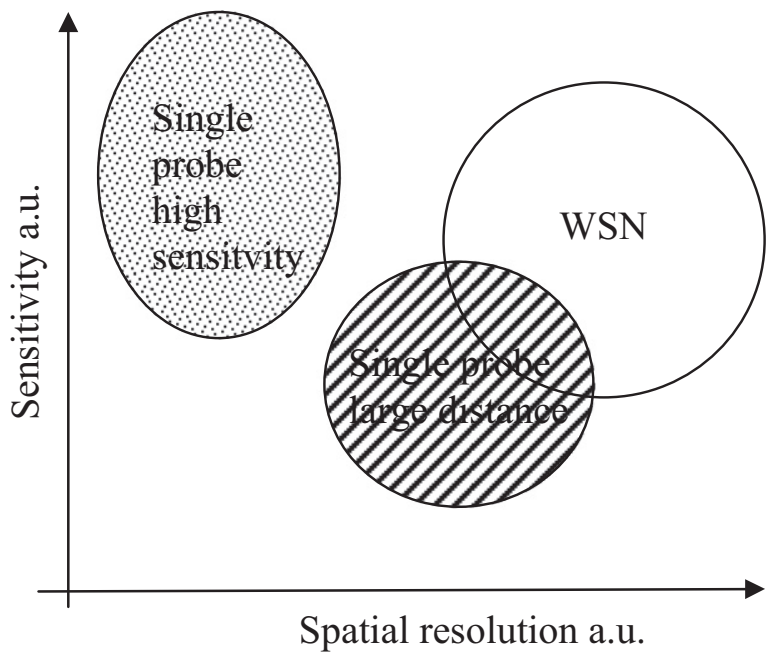

Fig. 1. Schematic showing the domain of application of WSN vs. single probe large distance measurements and single probe high sensitivity localized measurement.

(spectrometer for measuring soil, AFM, etc.), sensor capable of remotely mapping the atmosphere or the ground surface (LIDAR, telescope, atmospheric spectrometer, etc.), and/or simple local sensor (temperature, pressure sensor, etc).

Some large size single probes are capable of high quality local measurements with complex expensive instruments that have not yet been scaled down. Such type of measurements cannot for the moment be conducted by WSN.

WSN use essentially simple low cost sensors that are distributed in large numbers. In such scenario, if the position of each node is well known, the spatial resolution is excellent.

For WSN exploration missions, the robustness relies essentially on the whole network, i.e., nodes can fail without inducing a shut down of the whole network. Therefore, the testing procedures should be different compared to the ones for single probes based missions. Nodes would have to be tested in simulated harsh environments. Indeed, in WSN, due to the redundancy of the measurements, a higher node level of failure than for single node based mission could be tolerated. The whole WSN would have to be tested on Earth in scenarios as similar as possible to the ones encountered after deployment during the space mission.

\subsection{Economic benefits}

The costs of a mission based on WSN for space exploration depend on the objective, acceptable risk, and 
Table 1

Comparison of the scientific and technical aspects for one instrument based missions and ad hoc WSN based missions

\begin{tabular}{lll}
\hline Scientific and technical considerations & One instrument & Ad hoc WSN \\
\hline $\begin{array}{l}\text { Complex sensing involving long distance } \\
\text { measurements (spectrometry, LIDAR, opti- } \\
\text { cal, imaging) }\end{array}$ & Largely reported on past missions & Difficult due to: \\
& $\begin{array}{l}\text { Can obtain a large amount of data with } \\
\text { one single probe } \\
\text { The spatial resolution of those instru- } \\
\text { ments is a major limiting factor } \\
\text { Reported on past missions }\end{array}$ & • miniaturization problems \\
$\begin{array}{l}\text { Localized simple measurements (tempera- } \\
\text { ture, pressure, gas type and concentration, } \\
\text { humidity, light intensity) }\end{array}$ & $\begin{array}{l}\text { WSN bandwidth } \\
\text { Should enable mapping of the } \\
\text { parameters in a large area or } \\
\text { volume over a long period of } \\
\text { time if necessary }\end{array}$ \\
& $\begin{array}{l}\text { The data provided was for only one } \\
\text { single location on a planet or asteroid }\end{array}$ & Robust due to the AD-HOC network \\
\end{tabular}

qualification procedures. The cost of the hardware will be low, and dominated by development and qualification. The factors that can allow low costs for WSN based missions are simple testing procedure, small size and mass of the nodes and the possibility in the long term to use similar WSN equipment with different sensors, and the built-in redundancy that lowers the required reliability of each node.

\subsection{Benefits summary}

Compared to single probe missions, we can see many advantages in the long term with the introduction of WSN in space exploration:

- Shorter time between the elaboration of a mission and its launch.

- Simpler testing procedure.

- Higher reliability.

- Lighter payload.

- Lower costs.

The introduction of WSN in the space exploration domain will be gradual, starting with the development of a simple technology demonstrator mission.

\section{Alternative and potentially competing solutions to WSN}

There are three main ways to explore space:

- Using Earth based instruments.

- Single probe missions.

- WSN based missions.

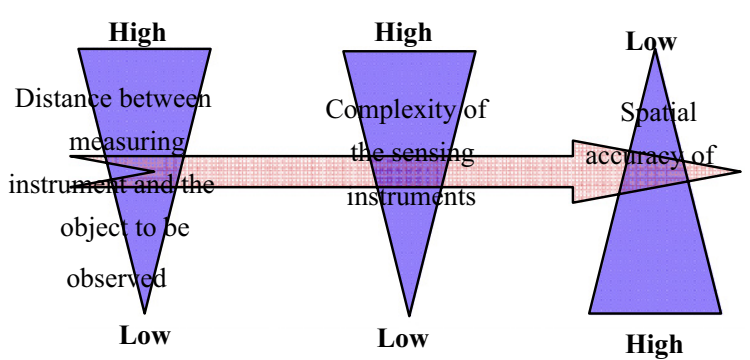

Fig. 2. Schematic showing the link between the instrument-object distance and the accuracy of the data measured.

Earth based instruments cannot map the Solar system to get the level of detailed information that can be obtained with exploration mission that are closer to the scientific data to be measured. Measuring with an instrument close to the object of interest provides much more accurate data than measuring from far away for two main reasons: the electromagnetic wave carrying the information can be modified by the physical channel it propagates through, and the signal-to-noise ratio (SNR) deteriorates with distance from the source. Placing the measuring instrument close to the source enables the use of simpler measurement techniques (Fig. 2). When we consider a network of instruments located close to the information sources, there is no need to map the environment around the instruments and the measurements are local.

Long or middle range distance measurements cannot provide as accurate data as numerous short distance measurements. When such a high spatial accuracy is not required, there can be some overlap between the different techniques (Fig. 1). 
The observation and exploration of the Solar system is maturing and scientists will want to obtain increasingly detailed data in order to map the atmosphere and ground of all the objects of the Solar system with high resolution.

\section{Environmental constraints}

The environmental constraints will vary largely from a mission to another:

Some example values:

- Temperature range: -133 to $+22^{\circ} \mathrm{C}$ on surface of Mars.

- Pressure range: up to 90 bar on the surface of Venus.

- Irradiation: krad to several mrad depending on orbit, Solar activity, and mission duration.

- Vibrations: up to $20 \mathrm{G}$ from 5 to $2000 \mathrm{~Hz}$ at launch.

- Shocks: up to $10,000 \mathrm{G}$ at separation of stages and heat shield.

Non-aerospace industry is developing WSN nodes capable to withstand harsh environment (for instance the European project e-CUBES [2]). These devices can be the starting point for optimizing nodes for specific missions.

\section{The operational constraints}

\subsection{Physical channel}

In many of the space exploration scenarios we have investigated, the overall footprint of the network can be up to $100 \times 100 \mathrm{~km}^{2}$. If nodes are only capable of communicating over a distance of a few $100 \mathrm{~m}$, this would largely limit the scenarios where WSN can be applicable.

The frequency band, type of modulation, emission power, and communication protocol strongly impact the performances of the WSN. Frequencies ranging from $1 \mathrm{MHz}$ to $10 \mathrm{GHz}$ would allow a good tradeoff between the antenna size and the communication distance that could be achieved in the presence of noise from external sources, i.e., galactic, Solar and atmospheric.

The power required to transmit in a defined channel is highly dependent on the distance between the transmitter and receiver. For a transmission in a defined physical channel that requires a transmission power $P_{t 1}$ for a distance $d_{1}$, a larger transmission power $P_{t 2}$ is required when the distance is increased at $d_{2}$ (see (1), Fig. 3 [3]). Depending on the physical channel characteristics, the power dependency or path loss (PL) factor can vary

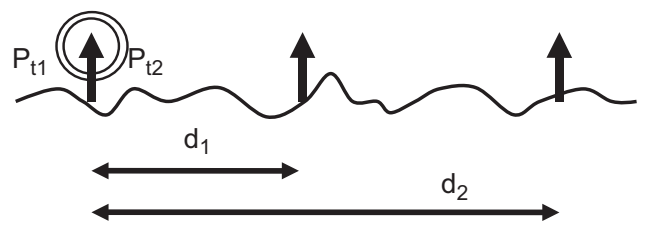

Fig. 3. Figure illustrating the power required to transmit at two different distances in the same physical channel.

from $n=2$ (in free space) up to $n=6$ (i.e., obstructed communication in building):

$P_{t 2}=P_{t 1}\left(\frac{d_{2}}{d_{1}}\right)^{n}$

All those aspect are tightly coupled and require a thorough study to optimize the WSN for any given scenario. A special challenge in WSN for space applications is the wide range of possible scenarios, each of which may have a very different RF solution.

\subsection{Power consumption issues}

One essential parameter for the success of WSN in space is power consumption [1]. All the options to achieve a low power consumption in a WSN have to be considered [4-6]:

- Dynamic power management/sleeping mode.

- Carrier frequency/antenna size and gain.

- Low power electronics.

- Modulation/multiple access techniques.

- Communication protocol/error control techniques.

Many scenarios for data acquisition by WSN in space exploration have a low sampling rate. Putting the nodes into "sleep" when not acquiring data is an excellent option to drastically reduce the power consumption. Different levels of sleeping mode could be considered depending on the scenario, the sampling rate and synchronization needs.

The antenna and selected carrier frequency also have a major influence on the overall performance of the nodes and their consumption. The gain of the antenna is related to its directivity. For a cloud of moving nodes distributed in the atmosphere omnidirectivity is desired and implies low gain antenna, while for nodes distributed in the same plane, higher gain antennas can be used. Moreover, the carrier frequency also influences the propagation losses or free space PL. For the same antenna dimensions and separation, the longer the carrier wavelength (the lower 
Table 2

Possible node distribution methods

\begin{tabular}{|c|c|c|c|}
\hline Distribution techniques & Advantages & Drawbacks & Typical mission \\
\hline \multirow{2}{*}{$\begin{array}{l}\text { Initial momentum (i.e., } \\
\text { explosive expulsion) }\end{array}$} & Simplicity & Could involve high accelerations & Atmospheric \& ground measurements \\
\hline & $\begin{array}{l}\text { Small node size } \\
\text { Could allow large distance } \\
\text { distribution }\end{array}$ & Not accurate distribution & \\
\hline Dropped from a spacecraft & $\begin{array}{l}\text { Simplicity } \\
\text { Small node size } \\
\text { Could allow large distance } \\
\text { distribution }\end{array}$ & $\begin{array}{l}\text { Could involve high accelerations } \\
\text { Not accurate distribution }\end{array}$ & Atmospheric \& ground measurements \\
\hline Distributed with a rover & $\begin{array}{l}\text { No need for node self localization } \\
\text { Small node size }\end{array}$ & $\begin{array}{l}\text { Large distance and node num- } \\
\text { ber distribution is time con- } \\
\text { suming }\end{array}$ & Ground measurements \\
\hline Individual propulsion & $\begin{array}{l}\text { Low accelerations } \\
\text { Could enable an accurate dis- } \\
\text { tribution }\end{array}$ & $\begin{array}{l}\text { Very complex } \\
\text { Large node size }\end{array}$ & Atmospheric \& ground measurements \\
\hline
\end{tabular}

the carrier frequency), the lower is the free space PL [7]. Thus, depending on the WSN scenario, different frequencies and types of antennas will have to be considered.

Additionally, using low power electronics impact the overall consumption of the WSN. Finally, the selection of an energy efficient modulation (rather than bandwidth efficient modulation) conjointly with the selection of an efficient multiple access technique and error coding scheme can also greatly contribute to minimize the overall power consumption.

\subsection{Energy source issues}

Depending on the mission and objectives, energy could be either harvested from the environment or/and stored in batteries. Energy harvesting being more complex than using batteries, the first WSN sent into space will be more reliable if using batteries.

Long duration mission will benefit most from harvesting technology. In the Solar system, the most common source of energy that can be harvested is sun light. Vibration energy or thermal gradients are much more difficult to harness.

\subsection{EMC issues with other systems in the environment}

In space exploration, normally there are no EMC perturbations coming from other systems in the environ- ment, other than the space craft. Furthermore, there are few limitations in the frequency band or emission power that can be used.

There have been some discussions regarding frequency allocation for space communication between NASA, ESA, and JAXA, but this imposes very few limits on WSN with communication ranges of only $100 \mathrm{~s}$ of $\mathrm{km}$.

\subsection{Localization of the sensors in the environment}

In most missions, localization of each node is essential in order to know where each measurement has been made. The node distribution method will strongly influence the localization technique [8] (Table 2). In some missions, nodes are moving relative to each other and therefore their position would have to be updated frequently, i.e., continuous localization.

Different localization techniques exist, but they rarely have been implemented on small size sensing nodes used in WSN and would have to be further developed. Table 3 gives an overview of localization techniques that could be used in space $[9,10,12-14]$. The localization process can be either intrinsic to the network, external or a combination.

For space exploration, the most promising localization techniques are based on pulsed LIDAR or RF waves. We evaluate these two promising techniques in Section 7. 
Table 3

Overview of localization techniques that could be used in WSN for space exploration

\begin{tabular}{|c|c|c|c|}
\hline Localization techniques & Implementations & Advantages & Drawbacks \\
\hline \multirow[t]{3}{*}{$\begin{array}{l}\text { RF electromagnetic wave } \\
\text { propagation delay }\end{array}$} & $\begin{array}{l}\text { Multilateration using time or time } \\
\text { difference of arrivals; phase or phase } \\
\text { difference of arrivals; round trip de- } \\
\text { lays }\end{array}$ & Can be very accurate & $\begin{array}{l}\text { Requires precise timing mea- } \\
\text { surements ( } 1 \mathrm{~ns} \text { error corre- } \\
\text { sponds to about } 30 \mathrm{~cm} \text { range } \\
\text { error) }\end{array}$ \\
\hline & & $\begin{array}{l}\text { Can be designed to } \\
\text { achieve long range mea- } \\
\text { surements }\end{array}$ & $\begin{array}{l}\text { Multipath propagation, i.e., } \\
\text { between nodes to nodes if } \\
\text { the nodes are located on the } \\
\text { ground, may result in large } \\
\text { measurement errors }\end{array}$ \\
\hline & & Can make use of UWB signals & $\begin{array}{l}\text { Phase measurements are sub- } \\
\text { ject to cycle slips and cycle } \\
\text { ambiguities }\end{array}$ \\
\hline RF signal strength & Assumes a given propagation model & Simple to implement & $\begin{array}{l}\text { May reduce the data rate } \\
\text { Very inaccurate when used } \\
\text { on ground due to signal fad- } \\
\text { ing caused by multipath (the } \\
\text { signal strength can vary by } \\
30-40 \mathrm{~dB} \text { over a distance of } \\
\text { half the wavelength of the } \\
\text { signal) }\end{array}$ \\
\hline \multirow[t]{2}{*}{ RF wave angle of arrival } & $\begin{array}{l}\text { Requires the use of at least two } \\
\text { antennas or a directional antenna } \\
\text { with known pattern }\end{array}$ & $\begin{array}{l}\text { The antenna array can be } \\
\text { installed at the BS only }\end{array}$ & $\begin{array}{l}\text { The accuracy is reduced with } \\
\text { Tx-Rx separation distance }\end{array}$ \\
\hline & & & $\begin{array}{l}\text { Possible ambiguities due to } \\
\text { multiple reflections (when the } \\
\text { nodes are located on the } \\
\text { ground) }\end{array}$ \\
\hline \multirow[t]{4}{*}{ Pulsed LIDAR } & Pulsed LASER on spacecraft/orbiter & $\begin{array}{l}\text { Can be very accurate } \\
(10 \mathrm{~m} \text { at } 10 \mathrm{~km} \text { distance })\end{array}$ & Limited to line of sight \\
\hline & Scanner on spacecraft/orbiter & $\begin{array}{l}\text { Distance of measurements } \\
\text { up to } 10 \mathrm{~km}\end{array}$ & $\begin{array}{l}\text { Probably limited on Mars to } \\
\text { of order } 10 \mathrm{~km} \text { with the exist- } \\
\text { ing technology to fit in mass } \\
\text { and power goals }\end{array}$ \\
\hline & Signal processing on spacecraft/orbiter & Independent of the data flow & $\begin{array}{l}\text { Larger payload on the space- } \\
\text { craft due to LIDAR equip- } \\
\text { ment }\end{array}$ \\
\hline & Corner-cubes reflector on nodes & & $\begin{array}{l}\text { Sampling time for whole scan } \\
\text { depends on the accuracy (di- } \\
\text { vergence) }\end{array}$ \\
\hline
\end{tabular}

\section{Proposed WSN for space exploration}

The proposed architecture of a WSN for space exploration is based on an ad hoc multi-hop network that collects scientific data and transmits it via a relay either directly to Earth or to an orbiter or spacecraft (Fig. 4). The topology of the network will largely depend on the type of mission, i.e., number of nodes, distance between the nodes, etc.

\subsection{Promising scenarios}

Depending on the exploration mission objectives, sensing nodes would have to face very different con- straints. Sensing nodes can be fixed either on the ground of a planet or asteroid or moving relative to each other. The nodes deployment technique would have a strong impact on the node network.

In a mission where nodes are falling through the atmosphere of a planet while taking measurements (Fig. 5), the relay for large distance transmission would fall among the nodes at the same speed. The duration of such a mission could be relatively short (up to a few hours) and the sampling rate quite high (for instance a data acquisition per second).

In a scenario where the sensing nodes land on the ground of a planet or moon (Fig. 6), a relay would transmit the data collected by the nodes to an orbiter 


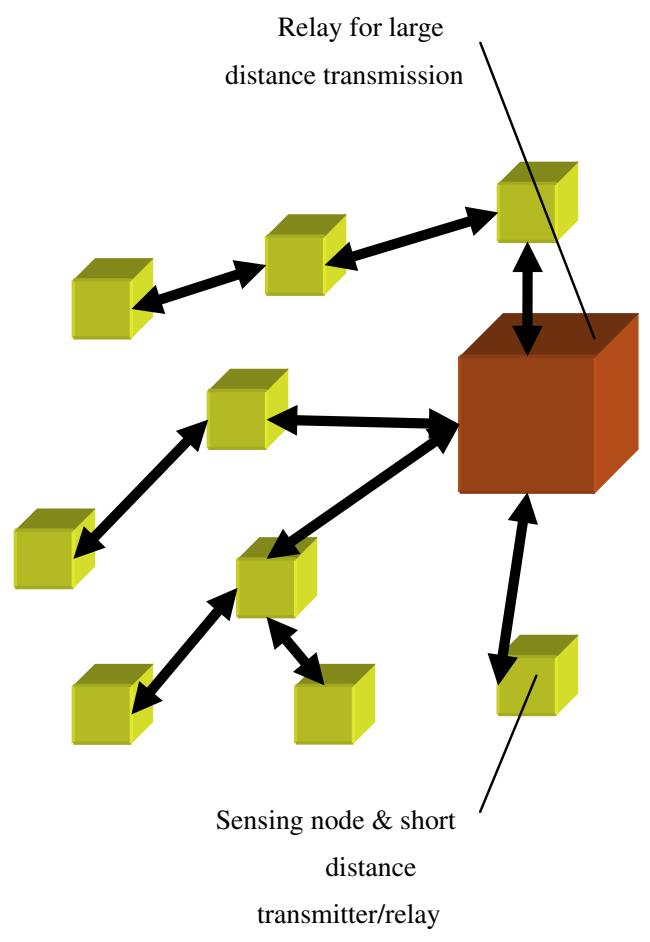

Fig. 4. Example architecture of a WSN used for space exploration.

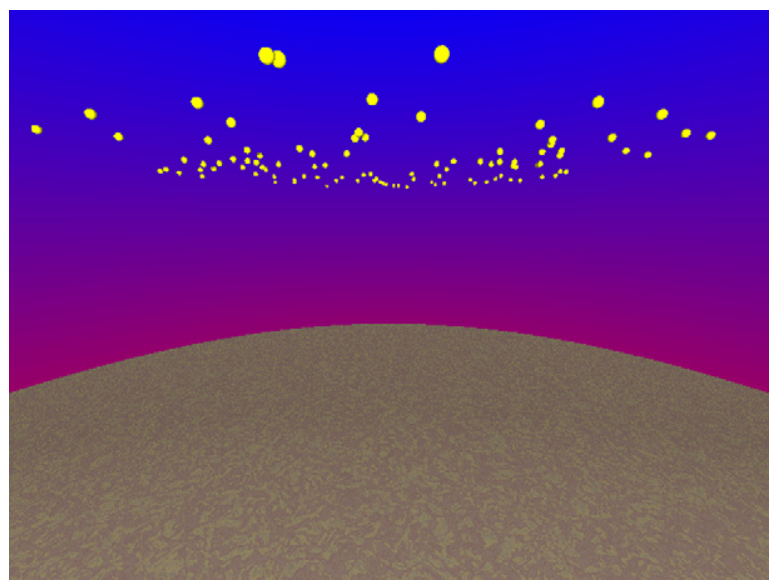

Fig. 5. Scenario of atmospheric measurements where the sensing nodes are falling through the atmosphere of a planet.

or directly to Earth. The mission could last up to a few years, while the sampling rate would be very low (for instance one data acquisition per hour). An alternative to this scenario for low mass Solar-system objects, i.e., asteroid, is to anchor the nodes into the ground for instance to conduct seismic measurements (Fig. 7).

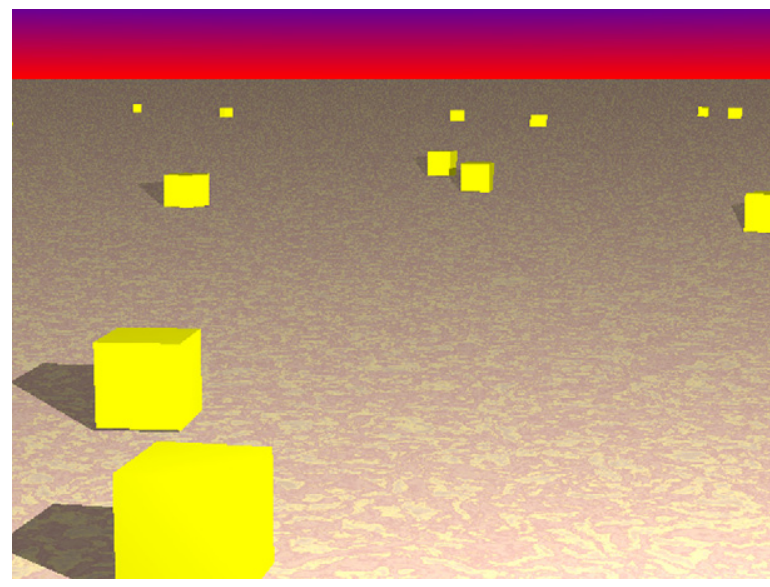

Fig. 6. Scenario where the sensing nodes are located on the ground of a planet or moon.

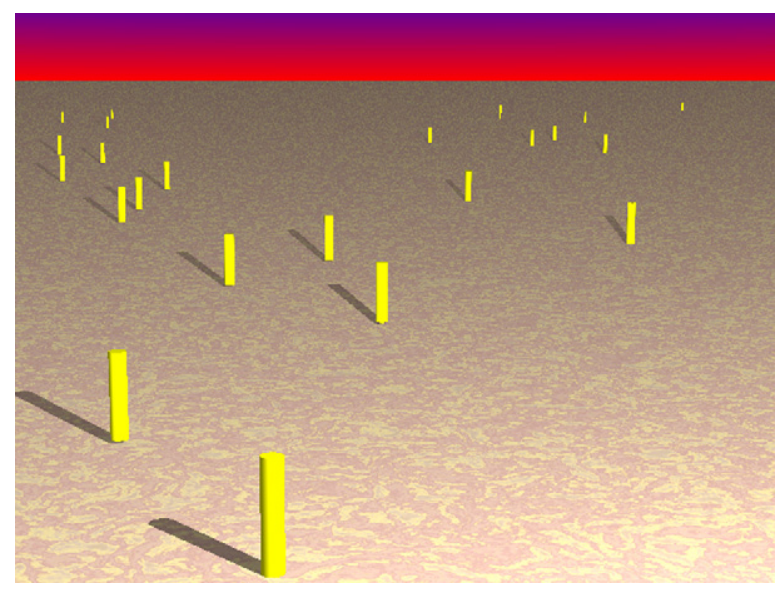

Fig. 7. Scenario where the nodes are anchored into the ground of an asteroid (low mass object).

More advanced scenarios would concern moving nodes over long period of time. For instance actively moving nodes, i.e., microrobot, on the ground of planets or moon could collect data while they would receive directions from Earth regarding the surface area to be explored. Dubowsky et al. proposed a concept where the nodes are rolling and bouncing on the ground [15]. In this perspective, different node design can be considered. For instance, the nodes could be constituted of a central heavy part that contains the electronics package into an inner sphere that is displaced by electroactive polymer actuators into an external hull. Slow motion of the actuators would induce a rolling behavior by continuously translating the center of gravity, while quick motion of the actuators would make the node jump over obstacles (Fig. 8). 


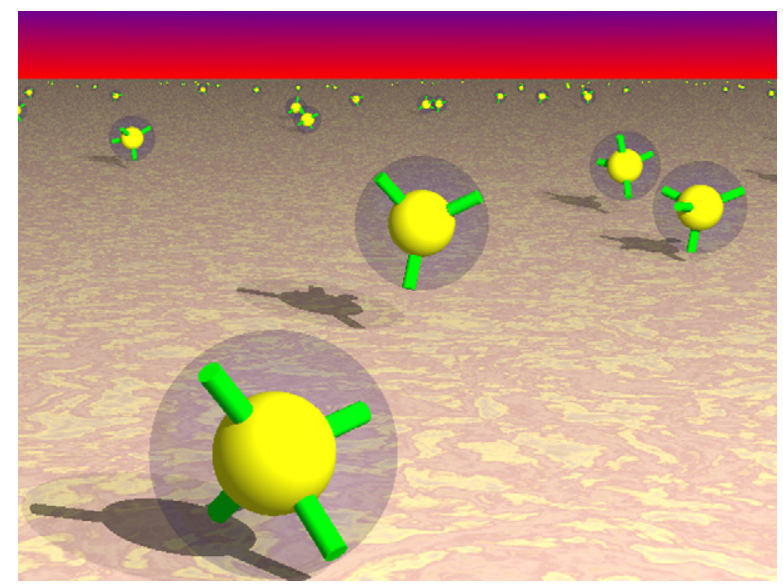

Fig. 8. Rolling and jumping nodes on the ground of a planet or moon.

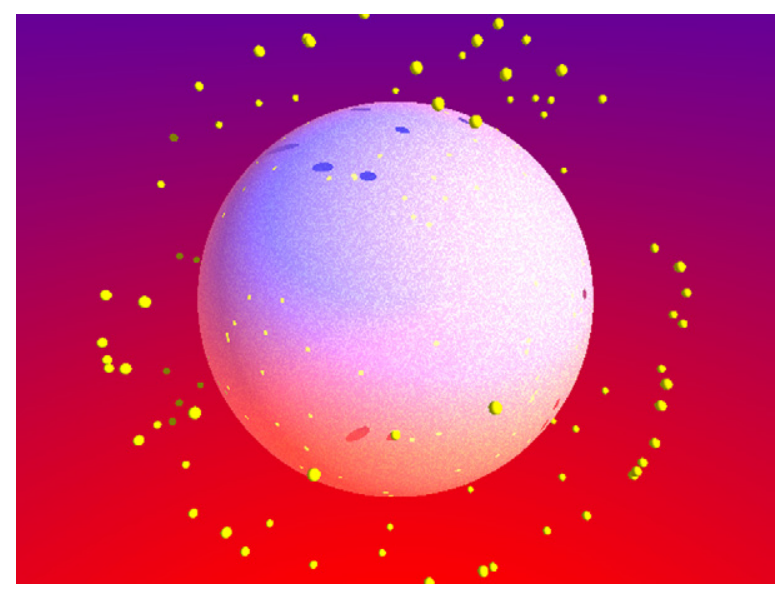

Fig. 9. Rebounding nodes around an asteroid (low mass object).

A more complex scenario could concern a cloud of nodes that would rebound on the surface [16] of a low mass object, i.e., asteroid (Fig. 9). The low attraction and the absence of gas would enable the nodes to rebound at high altitude (few kilometers) with relatively low impact speed. Acceleration sensors could provide data on the surface nature, for instance to locate a good landing place for large probes.

\subsection{Node architecture}

The nodes could have the following main components (Fig. 10):

- MEMS sensor (analog or digital).

- A to D converter (if analog sensors).

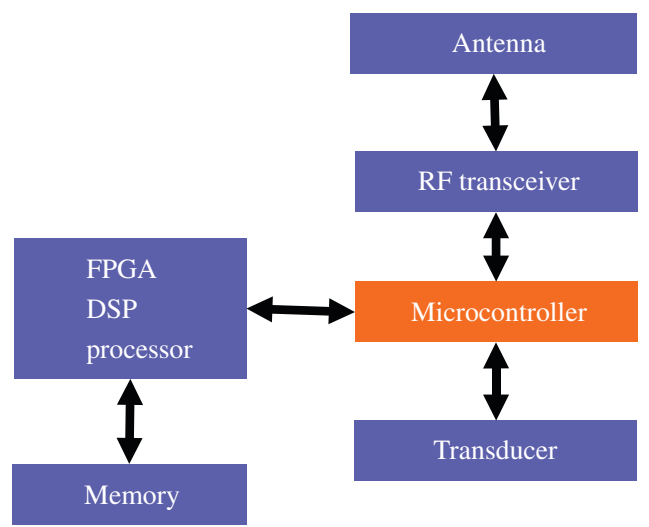

Fig. 10. Data flow between the different typical functional units.

- Microcontroller (signal conditioning, communication protocol and power management).

- DSP layer.

- Memory.

- RF transceiver.

- Antenna.

- Power supply.

In our view only the microprocessor can be common to all mission types. The other units would largely depend on the mission scenario, especially the RF components and sensors. Some parts may not be required in all cases, especially the DSP.

\subsection{The transducers}

The transducer section could typically consist of the following units (Fig. 10):

- MEMS sensor die for measuring for instance:

$\circ$ gas pressure;

$\circ$ temperature;

○ accelerations;

$\circ$ gas identification and concentration;

$\circ$ light intensity;

$\circ$ light direction.

- Signal conditioning IC with sensor functions and power supply voltage measurements.

The characteristics of the MEMS sensor would depend on the mission scenario and scientific objectives. Ideally, the microcontroller would be capable of conditioning different type of AC or DC signals from a variety of sensors. 


\subsection{The microcontroller}

The different level of software (OS, communication protocol, limited data analysis, localization calculations) would run on the microcontroller. Two options can be followed: either having the same high performance microcontroller for a variety of missions or using for each mission the minimal microcontroller in order to reduce power consumption.

\subsection{The RF transceiver}

The RF transceiver has to meet the requirements of a specific mission:

- emission power;

- frequency domain;

- drift of the oscillator over the required temperature and lifetime range;

- modulation and encoding scheme;

- localization;

- sleep mode;

- compatibility with the communication protocol;

- SNR;

- power consumption and efficiency;

- heat generation, etc.

Many of these parameters are tightly linked. The overall performance of the RF transmission would strongly depend on the interface to the antenna and the antenna. The interface between transceiver and antenna shall be as simple as possible, using a minimum of external components for the Rx/Tx switch and matching network.

\subsection{The antenna}

The mission type will influence the antenna choice:

- in free space the antenna has to be omni directional, or directional if coupled to a steering mechanism (mechanical or phased array);

- on the ground, the antenna would have to transmit in one plane, or focused if coupled to a steering mechanism.

Balanced antenna should be preferred to limit the influence of the ground while having a high gain. The antenna can be integrated into the nodes or unfolded/unrolled when the nodes are deployed. Probably a large effort would have to be placed in the development of compact antennas.

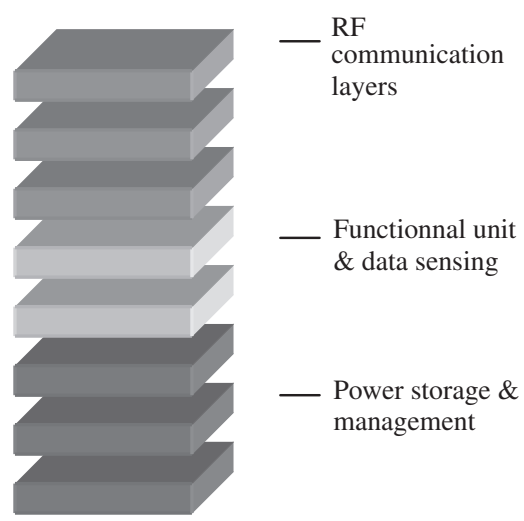

Fig. 11. In the e-CUBES packaging concept, nodes are constituted of a stack of all the functional layers in a volume of $1 \mathrm{~mm}^{3}$.

\subsection{Power supply system}

Depending on the mission and objectives, two types of energy source can be used:

- battery;

- energy harvesting (Solar energy).

Energy harvesting being more complex than using a battery, the first WSN sent into space would be more reliable if using a battery source that does not require DC/DC converters and charging circuitry.

\subsection{Packaging issues}

The packaging of the node must be robust and hermetic to allow for reliable operation on the asteroid, planet, or moon, as well as safe transit from Earth. The package serves as a mechanical support, and can provide electrical routing of signals and power.

For $\mathrm{cm}^{3}$ packages, ceramic chip carriers are an appealing solution, due to their robustness and also for instance to the ability to build a patch antenna directly into the lid. The EADS micropack [17] project is an excellent illustration of this concept, using a stack all functional layers, each in a ceramic package.

For $\mathrm{mm}^{3}$ packages, chip-scale and wafer-scale packaging becomes an important aspect, and integration of thin-film batteries and compact antenna will require novel packaging approaches. The European integrated project e-CUBES aims to integrate all the node elements into $1 \mathrm{~mm}^{3}$ (Fig. 11) using direct chip to chip stacking and bonding. Radiation shielding (most likely in the form of a few $\mathrm{mm}$ of $\mathrm{Al}$ ) may be required and may play a large role in node size and mass. 
Table 4

Example of specifications of WSN that could be used in the space exploration domain

\begin{tabular}{ll}
\hline Parameter & Value \\
\hline Range (distance between sensors) & $10 \mathrm{~m}$ to $10 \mathrm{~km}$ \\
Total network size & $500 \mathrm{~m}$ to $100 \mathrm{~km}$ \\
Sensor movement (in the network) & Fixed or mobile \\
Medium for wireless communications & Different gases, free space, surface of a planet \\
Volume occupied by all nodes while in transit & $\sim$ max $1 \mathrm{dm}^{3}$, the smaller the better \\
Lifetime & Under operation: hours to few years \\
Frequency range for communications & $1 \mathrm{MHz}$ to $\sim 10 \mathrm{GHz}$ \\
Type of data transfer & Continuous, duty-cycled or occasional \\
Data rate provided by a sensor & Few byte per acquisition \\
Temperature range & For instance -140 to $+30^{\circ} \mathrm{C}$ on the surface of Mars \\
Main available energy from the environment & Solar \\
Need for network self-organization & Yes, and continuous in case some node fails or are mobile \\
Need for a localization of the nodes & Yes, at high rate if the network is mobile \\
Typical transmit power (can be largely dependant on the & $10 \mathrm{~mW}$ when transmitting, could be lower \\
mission and technology) & \\
Typical total energy for a mission per node & Typically $1 \mathrm{Wh}$ for one year operation at a rate of $10 \mathrm{~min}$ \\
& operation per day during one year
\end{tabular}

\subsection{Software}

Minimizing the software is an interesting option that reduces power consumption and hardware requirements.

The software functionalities would largely depend on the mission scenario. There can be three levels of software:

- Upper level programs for data processing.

- Layer that handles the data flow and the power management.

- The communication protocol (medium access control, MAC, level).

An OS can be used to support the upper level programs and facilitate the data flow management.

The main functionalities of the software that are particular to the WSN used for space exploration are:

- Sleep mode: different sleeping mode can be considered in order to reduce consumption during operation and minimize EMC perturbations during node distribution.

- RF localization calculations: can be based on timeof-flight or amplitude in order to map the topology of the network.

\subsection{Specifications of visionary demonstrators}

In Table 4 typical specifications for space exploration with WSN are summarized.

\section{Evaluation of localization techniques}

The characteristics of WSN would have to vary greatly from one mission to another. Nevertheless, we tried to define the typical requirements for a WSN used for space exploration (Table 4). Implications and tradeoffs of localization techniques are thus best illustrated when considering specific exploration scenarios.

We evaluate two approaches to locate nodes based on LIDAR or on RF electromagnetic waves. Since localization is highly mission dependent, we consider two specific exploration scenarios that illustrate the requirements and performances of each technique.

\subsection{Evaluation of a LIDAR based localization technique during a mission to Mars}

For on the surface measurements, we investigate the feasibility of the following scenario for locating nodes:

We suppose that the nodes are dispersed by a spacecraft/lander. After their dispersion they are free falling towards the planet's surface. The spacecraft/lander may maneuver, i.e., it is not descending in free-fall. In this way, the nodes, after some time are well below the spacecraft/lander and reach the surface before it (Fig. 12).

We assume a LIDAR (laser radar) observing the planet's surface and the cluster of free-falling nodes. The assumed LIDAR is a "time-of-flight" with multiple start-stop, i.e., with the possibility to observe several targets during one pulse swap [18]. The LIDAR is equipped with a scanner, allowing the scanning of 


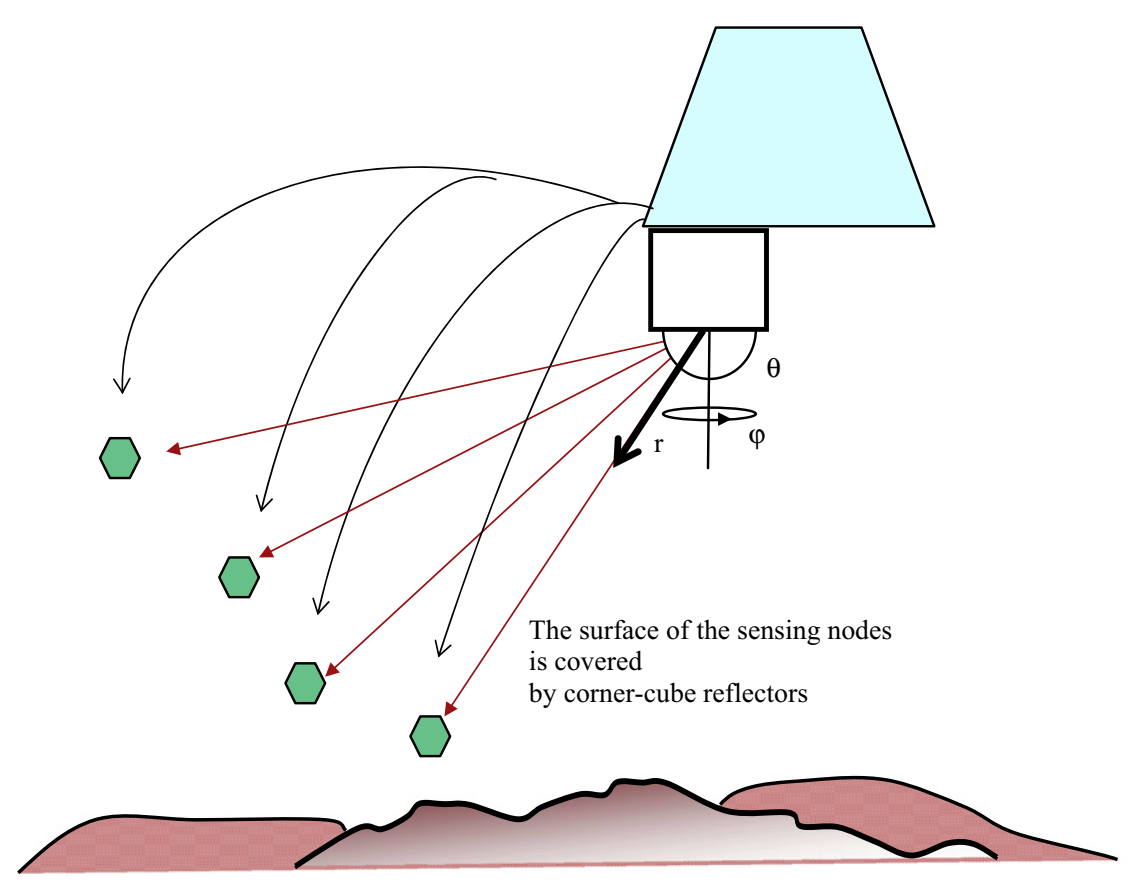

Fig. 12. Scenario proposed for LIDAR localization technique evaluation: a spacecraft drops the sensing nodes a few $10 \mathrm{~s}$ of kilometers above Mars' surface. The spacecraft is equipped with a LIDAR. The nodes are not shadowed from the spacecraft by terrain features.

the laser beam together with the receiver field-of-view (FOW) of the receiver. This scanning shall be fast, which may be achieved if the detection of the node is feasible with one laser pulse only. In this way the LIDAR measurement locates the node, i.e., determines the range from the LIDAR time-of-flight measurement and the angles of elevation and azimuth from the scanner position.

The requirement to the LIDAR is to detect the node with one-laser pulse. This feasibility study is carried out by numerical simulations of the LIDAR detection performances, with the following inputs: specifications of the LIDAR subsystems, the specifications of the nodes, the characteristics of the planet's environment relevant to the detection. The output is the SNR for detection of the back-reflected signal from the node, where the detection is considered successful with SNR $>2$.

\subsubsection{Conditions}

We have investigated several LIDAR configurations and present some results obtained with one of the LIDAR operating at $532 \mathrm{~nm}$ (with the second harmonic of a microchip Nd:YAG laser). The other principle subsystems specifications of this LIDAR are given in Table 5.

The nodes have a Polyhedron-type shape, where on each wall is attached a layer of corner-cube reflectors,
Table 5

Localization with LIDAR, considered subsystems specifications

\begin{tabular}{ll}
\hline Pulse repetition rate & $10 \mathrm{kHz}$ \\
Laser pulse energy & $1 \mu \mathrm{J}$ \\
Beam divergence & $1 \mathrm{mrad}$ \\
Receiver telescope diameter & $50 \mathrm{~mm}$ \\
$\begin{array}{l}\text { Receiver field-of-view } \\
\text { Full-width at half-maximum }\end{array}$ & $2 \mathrm{mrad}$ \\
(FWHM) of the interference filter & $0.3 \mathrm{~nm}$ \\
$\begin{array}{l}\text { Overall end-to-end optical efficiency } \\
\text { (incl. the filter transmission) }\end{array}$ & 0.1 \\
$\begin{array}{l}\text { Quantum efficiency of the detector } \\
\text { Detector dark noise equivalent to }\end{array}$ & 0.4 \\
\hline
\end{tabular}

having reflective surface of $1 \mathrm{~cm}^{2}$ and back reflectivity efficiency better than 0.3 . In the performance simulations the nodes dispersion is assumed to be on the surface of Mars, day-time. The attenuation of the Mars atmosphere is caused by the airborne dust, where we take a simplified model for its distribution and optical attenuation. Three values of atmospheric optical depth (OD) are assumed to be $0.1,0.5$ and 2.0. The layer of airborne dust is taken with a homogeneous altitude distribution between the surface and $15 \mathrm{~km}$ altitude. Although simplified and approximate, such values are compatible with the Mars atmospheric models and observations $[19,20]$. 


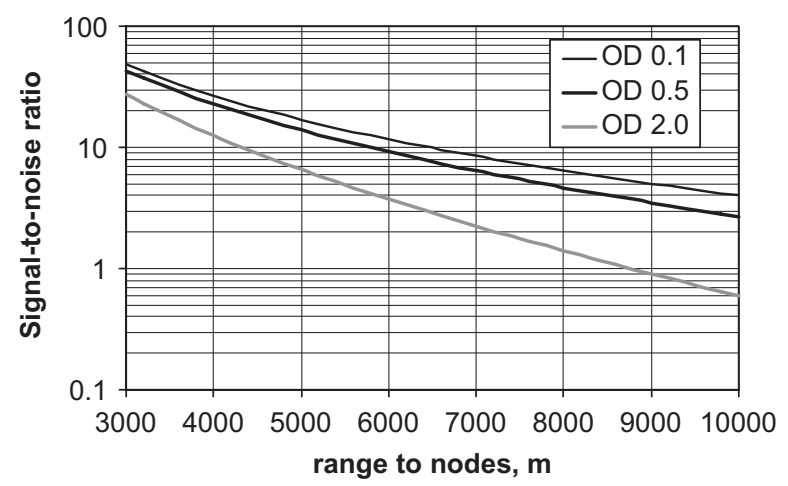

Fig. 13. Signal-to-noise ratio for detection of a single light pulse retroreflected by the node and received by the detector in the spacecraft. This light pulse was reflected by the node situated at a certain range from the spacecraft. For signal-to-noise ratio above 2, the detection is considered successful. The detection takes place with nodes and spacecraft below $10 \mathrm{~km}$ altitude above surface level.

\subsubsection{Localization and detection simulations}

Fig. 13 presents simulations of the SNR for nodes detection in one laser pulse only, as a function of the range to the node, for the three selected values of the atmospheric OD. As we may expect, the SNR values decrease with OD increase. Nevertheless, even for the highest assumed OD, the one-shot detection is feasible at ranges till $7000 \mathrm{~m}$ from the LIDAR (spacecraft).

We have also calculated the LIDAR performance for Mars surface detection, using a surface albedo 0.3. As expected, the range to obtain $\mathrm{SNR}=2$ is shorter than for nodes location, since the signal is backreflected not by a retroreflector, but by a Lambertian surface. Nevertheless, the results show that a SNR $>2$ may be obtained for ranges below $2500 \mathrm{~m}$. I.e., the same LIDAR used for nodes detection may be also used for surface imaging with objectives surface topography and selecting landing site.

Once the nodes have reached the ground, the much higher strength signal they reflect than the one reflected by the ground, i.e., about 100 times stronger than the response from the surrounding Lambertian surface, allows to locate them.

\subsubsection{Scanning time considerations}

The scanning time is dependant on the sampling rate and the divergence. If we consider a divergence of $1 \mathrm{mrad}$, which corresponds to a spatial accuracy of $10 \mathrm{~m}$ at $10 \mathrm{~km}$ distance, to scan $1 \mathrm{rad}$ in two orthogonal directions, it would take $100 \mathrm{~s}$. Such a time is acceptable to locate static nodes but unrealistic for a dynamic scenario where nodes are moving in space or atmosphere. There are ways to reduce the scanning time: increasing the divergence, reducing the scanned volume, using a brush like laser beam, or using an imaging laser detector. Probably scanning with 2-D beam pattern having for instance $100 \times 100$ beams is a promising approach that would allow a substantial scanning time reduction; this approach would eventually be fast enough to locate moving nodes.

The 2-D measurements may be carried by scanning the laser beam only. The detection of the back reflected signal from different directions may be performed by a 2-D optical detector in photon-counting mode (SPAD) [21].

\subsubsection{Identification of nodes}

To measure valuable data with the sensing network, it is necessary to correlate sensed data with node location. We propose to use electronic tags activated by the LIDAR to identify each node.

It is assumed that during the identification the spacecraft is sufficiently above the surface, while the nodes have already landed. Each node would have a light sensor sensitive to the LIDAR pulse. When a node is interrogated by the LIDAR signal, it would send its identification code (node tag) to the base station, either with a light signal observed by the LIDAR receiver (Fig. 14) or through the RF data flow with in addition the time it was interrogated by the LIDAR. For this purpose an optical communication "mini-terminal" is installed on the node containing a receiver ("listener") and a transmitter ("responder") (Fig. 14).

The optical receiver of the "listener" is based on: a miniature lens having sufficiently large FOV (ultimately "all-sky" lens), optical filter, avalanche photodiode (APD), as well as the respective signal detection and recognition electronics. In the identification concept where the LIDAR operates as optical communication terminal, the light transmitter would be based on the same type of lens, light-emitting diode (LED) and the electronic circuit forming the node tag and driving the LED.

Numerical simulations were performed to evaluate the SNR for recognizing the LIDAR questioned by the "listener" and to recognize the answer from the "responder" by the LIDAR receiver. For numerical simulations, most LIDAR transmitter specifications remain unchanged. The new parameters related to the operation as optical communication "mini-terminal" are presented in Table 6. The considered specifications of the "responder" for simulation are presented in Table 7.

Fig. 15 presents the results from numerical performance simulation for the two steps of the optical based nodes identification. The simulation results with 

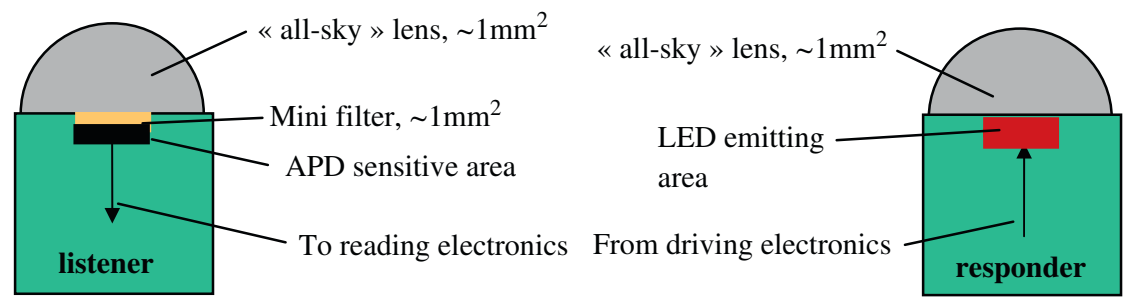

Fig. 14. Node identification concept based on optical mini-terminal located in each node.

Table 6

Identification with Lidar, LIDAR specifications with listener/responder added constrains

\begin{tabular}{ll}
\hline Quantum efficiency (at wavelength $800 \mathrm{~nm}$ ) & 0.6 \\
Overall transmission & 0.2 \\
Optical filter bandwidth, to fit to the & $80 \mathrm{~nm}$ \\
bandwidth of the LED radiation & $1 \mathrm{~mm}^{2}$ \\
Receiver diameter & $20 \mathrm{~nm}$ \\
Interference filter FWHM & 0.6 \\
Interference filter transmission & \\
\hline
\end{tabular}

Table 7

Identification with LIDAR, specifications used in simulations

\begin{tabular}{ll}
\hline LED central wavelength & $800 \mathrm{~nm}$ \\
Pulse repetition rate & $25 \mathrm{MHz}$ \\
Pulse duration & $20 \mathrm{~ns}$ \\
Pulse energy & $40 \mathrm{~nJ}$ \\
Answer word length & 512 -bit (50\% duty cycle) \\
$\begin{array}{l}\text { Night-time, OD } \\
\text { No. of interrogating signal laser } \\
\text { pulses }\end{array}$ & 2 \\
$\begin{array}{l}\text { No. of repetition of the answering } \\
\text { sequence }\end{array}$ & 3 \\
$\begin{array}{l}\text { Total number of optical pulses in } \\
\text { the response }\end{array}$ & 40 \\
Transmission duration & 10,240 \\
\hline
\end{tabular}

LED-based "responder" confirm the feasibility of this method during night-time for distances up to $7 \mathrm{~km}$. We have also performed performance simulations with laser diode-based "responder" and, respectively, optical filter of the LIDAR receiver $1 \mathrm{~nm}$ FWHM. These simulations show that detection of the responder signal may be possible also at daytime with the same time duration as the one shown in Fig. 15, but for ranges to nodes below $2200 \mathrm{~m}$.

As we may see, the identification by optical responder is possible, but requires a technical complication of the LIDAR and the nodes, as well as a separate mode for beam-scanning, inducing a longer time to complete the process.

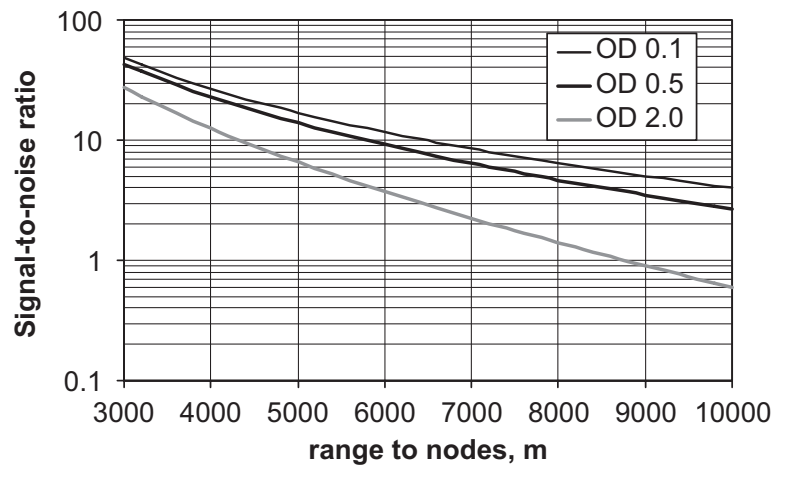

Fig. 15. Signal-to-noise ratio for detection of the questioning pulse sequence from the "listener" and the "answering sequence" from the LIDAR receiver in questioning mode; the simulation is for night time on Mars.

\subsubsection{LIDAR for localization and identification summary}

The numerical performance simulations demonstrate the feasibility to use LIDAR for locating nodes. Even for the case of Mars, with its attenuating atmosphere, such localization calls for LIDAR specifications that can be met with the present technology for compact lasers and detectors. The requirements on the LIDAR scanner may be quite critical.

Such LIDAR may be also used as a terminal in identifying nodes by a simple optical communication link. This requires a reasonable complication of the nodes by adding a mini-terminal for free-space optical communication, containing at least a "listener". The identification tag could be sent either optically ("responder") or through the RF channel.

One must note that when using LIDAR for localization, the nodes shall be not shadowed by terrain features from the LIDAR.

\subsection{Evaluation of RF based localization technique}

For atmospheric measurements, we investigate the feasibility of the following scenario for locating nodes: 

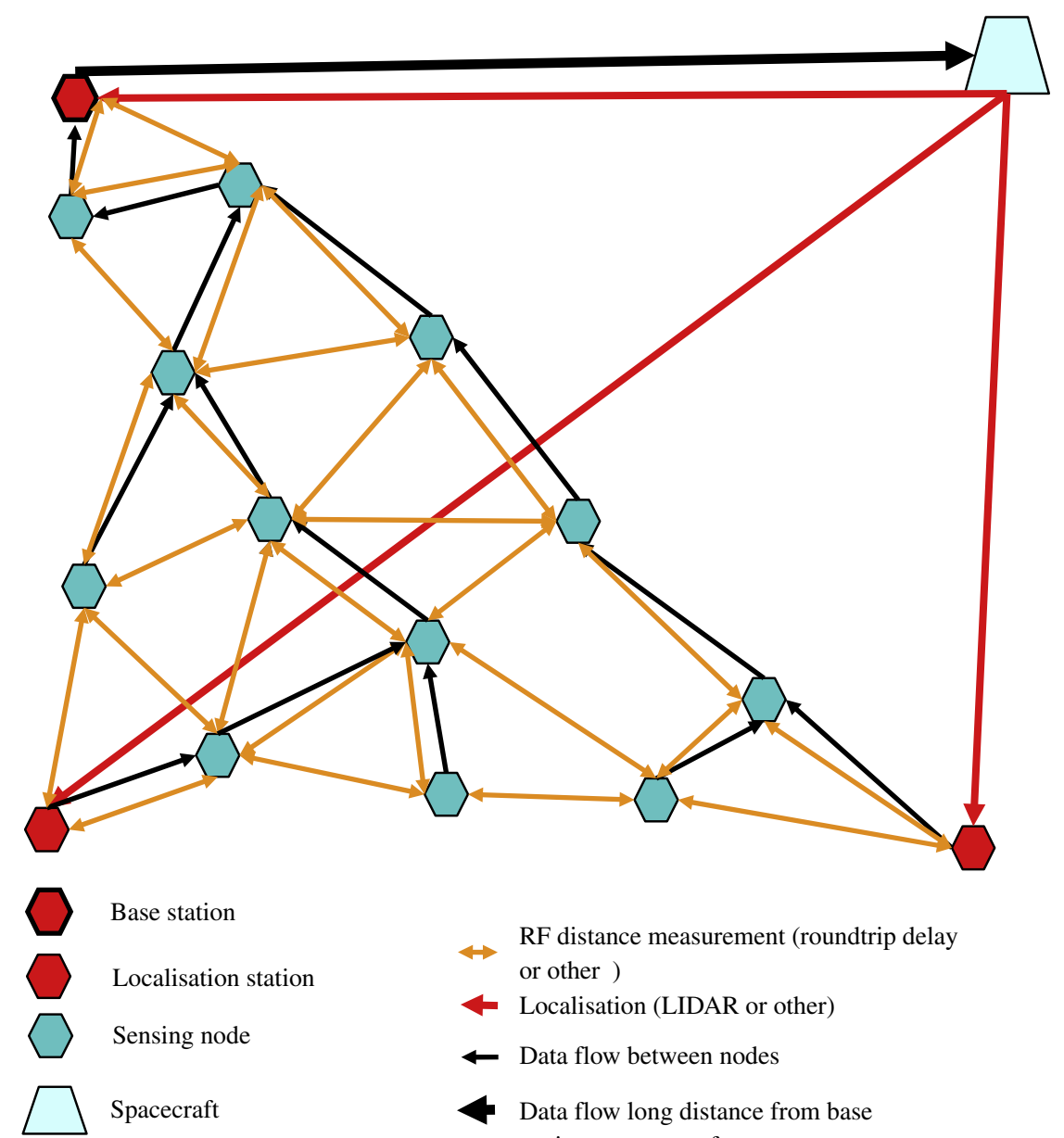

$$
\begin{aligned}
& \leftrightarrow \quad \begin{array}{l}
\text { RF distance measurement (roundtrip delay } \\
\text { or other ) }
\end{array} \\
& \leftarrow \text { Localisation (LIDAR or other) } \\
& \leftarrow \text { Data flow between nodes } \\
& \leftarrow \begin{array}{l}
\text { Data flow long distance from base } \\
\text { station to spacecraft }
\end{array}
\end{aligned}
$$

Fig. 16. Schematic of a typical network implemented with RF based localization techniques.

We suppose that the nodes are dispersed by a spacecraft orbiting around a planet. After their dispersion they are distributed as a node cloud moving through atmosphere. The spacecraft may maneuver and follow the cloud. For the localization of the sensing nodes we evaluate different RF options based on round trip delay, signal strength and angle of arrival. To locate the sensing nodes within the network, the network has to be referenced to the spacecraft. In the case where only inter-node distance measurements are considered, the position of at least three nodes has to be referenced to the spacecraft [10], if the angle of arrival information is present then only one node has to be referenced. A way to locate these three nodes constantly is to track them with a LIDAR (Fig. 16).

For estimating the node location with different RF based localization techniques, we discuss the Cramer-Rao lower bound (CRLB), which provides a lower limit of the best accuracy any unbiased estimator may provide [10], and thus provides a benchmark to compare the different techniques.

\subsubsection{Localization techniques based on RF electromagnetic waves propagation delay}

In [22], the CRLB for the estimation of the time-delay of a signal $s(t)$ band limited to $\pm B \mathrm{~Hz}$ in additive with additive white Gaussian noise (AWGN) was derived and given as (2)

$$
\operatorname{var}(\hat{\tau}) \geqslant \frac{1}{\operatorname{SNR} \overline{F^{2}}}
$$

where SNR is the received signal-to-noise ratio and $\overline{F^{2}}$ is the mean-square bandwidth of the signal given by (3)

$\overline{F^{2}}=\frac{\int_{-\infty}^{\infty}(2 \pi f)^{2}|S(f)|^{2} \mathrm{~d} f}{\int_{-\infty}^{\infty}|S(f)|^{2} \mathrm{~d} f}$

and $S(f)$ is the Fourier transform of $s(t)$. 
Since $\overline{F^{2}}$ depends on the signal's pulse shape, we consider as an example a Nyquist or sinc pulse for which $\overline{F^{2}}=\frac{4}{3} \pi^{2} B^{2}$. In this case, the CRLB for the estimation of the time-delay becomes (4)

$\operatorname{var}(\hat{\tau}) \geqslant \frac{0.076}{\operatorname{SNR} \bar{B}^{2}}$

We see that the time-delay accuracy (i.e., its standard deviation or the square root of the variance $\operatorname{var}(\hat{\tau}))$ is inversely proportional to the bandwidth of the signal. For example, if the signal's bandwidth is $1 \mathrm{MHz}$, the CRLB from the range estimation $c \hat{\tau}$, where $c$ is the speed of light, is (5)

$\operatorname{var}(c \hat{\tau}) \geqslant \frac{82.7}{\mathrm{SNR}}$.

That is, for a $1 \mathrm{MHz}$ signal and a $10 \mathrm{~dB}$ SNR, the best achievable accuracy using a sinc pulse shape is $2.876 \mathrm{~m}$. On the other hand, using a $1 \mathrm{GHz}$ signal bandwidth, the best achievable accuracy becomes $2.876 \mathrm{~mm}$. Note that in the presence of RF reflectors such as when the nodes are located on the ground, the above accuracy will not likely be achievable for narrowband signals due to multipath interference. In this case, ultra wideband (UWB) signals can be used to reduce the effects of multipath and achieve a better accuracy $[10,14]$.

\subsubsection{Localization techniques based on received signal strength (RSS)}

In [22], the CRLB for the estimation of the received signal strength (RSS) of a sinusoidal signal of amplitude $A$ embedded in AWGN with variance $\sigma^{2}$ and using $N$ uncorrelated samples was derived and given as (6)

$\operatorname{var}(\hat{A}) \geqslant \frac{2 \sigma^{2}}{N}$

Since in free space the received power $\operatorname{Pr}(d)$ at a distance $d$ from the transmitter will follow Friis formula and decrease as a function of the square of the distance $d$, we can write (7)

$A^{2} \propto \operatorname{Pr}(d) \propto K / d^{2}$

where $K$ is a constant that depends on the transmitted power $P_{t}$, the transmitter and receiver's antenna gains $G_{t}$ and $G_{r}$, respectively, and on the signal's wavelength $\lambda$, and is given as (8)

$K=\frac{P_{t} G_{t} G_{r} \lambda^{2}}{(4 \pi)^{2}}$

Using the relation $d=K^{\prime} / A$ (see (7)), we can thus calculate the CRLB for the range estimation from the
CRLB for the amplitude estimation as (9)

$\operatorname{var}(\hat{d}) \geqslant\left[\frac{\partial d}{\partial A}\right]^{2}, \quad \operatorname{var}(\hat{A})=\frac{d^{2}}{\mathrm{SNR} \times N}$

We see that the range accuracy (i.e., the square root of the variance $\operatorname{var}(\hat{d})$ ) for a given received SNR will decrease, i.e., its standard deviation will increase, as the distance increases, contrary to time-delay based range estimation which is independent of the distance assuming a constant received SNR.

As an example, for a $10 \mathrm{~dB}$ SNR, 100 independent measurements, and a distance $d=10 \mathrm{~m}$, the best achievable accuracy assuming free-space conditions is about $32 \mathrm{~cm}$. On the other hand, for a distance of $1 \mathrm{~km}$ and the same other conditions, it becomes $31.6 \mathrm{~m}$ and even $316 \mathrm{~m}$ for $d=10 \mathrm{~km}$, which is certainly not sufficient for locating the nodes in a scientific mission. In addition, even for scenarios where the separation distance between the nodes would be limited (e.g., to less than $1 \mathrm{~km}$ ), there are other considerations that may still prohibit or limit the use of a RSS-based localization technique:

- Ideally, for 3-D positioning, the transmitter and receiver antennas should be isotropic. However, in practice, 3-D isotropic antennas are difficult to realize. Since for space exploration, the transmitter and receiver antennas may spin independently in any direction, more than one antenna could be used and combined together at the receiver to compensate for its non-perfectly isotropic antenna patterns. However, compensating for the antenna pattern of the transmitter is not so obvious as it could be pointing in any direction (note that $\mathrm{a} \pm 3 \mathrm{~dB}$ difference in antenna pattern will result in a range estimation error by a factor of $\sqrt{2}$ ).

- In non-free space conditions such as on the ground in a multipath fading environment, the variations in the signal strength can be as great as $30-40 \mathrm{~dB}$ over distances on the order of half the wavelength of the signal. In addition, other environmental factors such as dust can affect the signal.

- The amplitude of the transmitted signal must be known at the receiver and may depend on the battery level (note that it could be measured at the transmitter and transmitted to the receiver).

\subsubsection{Localization technique based on angle of arrival and/or departure}

In this case, it can also be easily verified [23] that the range accuracy for a given received SNR will decrease 
as the distance increases, similarly to the RSS localization technique discussed above. This means that for compensating relatively long RF propagation distances, a relatively large array of antenna elements will have to be used in order to provide a sufficiently good angle of arrival and/or departure estimation and in turns a sufficient positioning accuracy (for a linear array geometry, doubling the number of antenna elements will reduce the variance by a factor of approximately 8 [22]).

Note that while a sufficiently large array may be prohibitive for its implementation on the sensing nodes, it could be implemented on the relays or on the spacecraft observing the nodes free-fall.

\subsubsection{Other practical considerations}

Minimum transmitted power: The minimum transmitted power will depend on many factors such as the distance between transmitter and receiver, the antennas, the carrier frequency and signal bandwidth, the atmosphere attenuation and the modulation scheme. Typically, for a $2.4 \mathrm{GHz}$ carrier frequency and isotropic antennas, the free-space PL will be given by $10 \log \left(\lambda^{2} /(4 \pi)^{2} d^{2}\right)$ or about $-120 \mathrm{~dB}$ for $10 \mathrm{~km}$. If the receiver bandwidth $\mathrm{B}$ and noise figure $\mathrm{NF}$ are $100 \mathrm{kHz}$ and $3 \mathrm{~dB}$, respectively, and a SNR of $10 \mathrm{~dB}$ is desired, then the minimum transmit power in $\mathrm{dBm}$ will be $\mathrm{SNR}-\mathrm{PL}+\mathrm{NF}+$ $10 \log (k \mathrm{~TB})=10+120+3-174+10 \log \left(1 e^{5}\right)=9 \mathrm{dBm}$ or $7.9 \mathrm{~mW}$.

Minimum quartz accuracy: In order to limit the required quartz accuracy, it is possible to estimate the relative round trip delay of a signal between any pair of adjacent sensing nodes instead of the absolute time of arrivals. In this case, the clock accuracy should be such that during a round trip delay the clock deviation is small. For a distance of $10 \mathrm{~km}$, the propagation time is roughly $33 \mu \mathrm{s}$. Assuming that the delay in the receiver prior to a retransmission is on the order of a few tens of $\mu \mathrm{s}$, the total round trip delay will be less than $100 \mu \mathrm{s}$. Thus, in order to minimize the error due to the quartz stability to less than $30 \mathrm{~cm}$ or $1 \mathrm{~ns}$, the quartz stability should be in the order of $10 \mathrm{ppm}$, which is easily achievable for a temperature control crystal oscillator (TCXO).

In addition, for a system where the positions of the nodes are calculated remotely on a central processor (e.g., located on the spacecraft) based on the individual and asynchronous nodes measurements, it is desired that every node making a range measurement with another node will timestamp its measurement as the measurement may transit a non-deterministic time through the $\mathrm{WN}$ prior to be received by the central processor, i.e., if the nodes are free-falling for $20 \mathrm{~min}$ and a $10 \mathrm{ppm}$ quartz is used, the clock error could reach $12 \mathrm{~ms}$. However, we see that this is not a too critical issue as a typical descent velocity in the atmosphere of a planet would be below $100 \mathrm{~m} / \mathrm{s}$ corresponding to a positioning error limited to only $1.2 \mathrm{~m}$. Note that time stamping the nodes measurements has the additional advantage that it becomes possible for the remote location processor to interpolate between the asynchronous measurements and thus obtain time synchronous measurements snapshots.

Algorithms for position estimation in a WSN: The localization algorithms can be implemented locally in each node (distributed algorithm) or concentrated in one node (centralized algorithms), i.e., the base station [10]. In an exploration mission where nodes are moving freely without directed motion toward a specific destination, there is no need for nodes to know their own position. Therefore, we think that centralized algorithms are more appropriate. Numerous studies compare different types of localization algorithms in terms of energy efficiency and accuracy $[10,12,13]$. For space exploration, the best suited algorithm would certainly depend on the mission scenario.

\subsection{Localization summary}

RF based and LIDAR based localization techniques can be complementary. LIDAR works only in line of sight and is easier to implement when nodes are either static or moving slowly, and can also be used when nodes are located on the ground of a planet. However, tracking accurately with a LIDAR the location of numerous moving nodes is a challenging task to realize with accuracy, and it is thus more realistic considering today's technology to track only a limited number of nodes. On the other hand, RF based localization techniques can be used to locate numerous nodes distributed in free space even when they are moving fast relative to each other for instance in a cloud formation. In particular, RF localization based on round trip delay measurements presents the best compromise between accuracy and complexity as compared to other RF techniques, but requires having at least three location reference nodes [10]. A measurement technique that combines round trip delay and angle of arrival (LIDAR or RF based) can thus provide the location reference for these reference nodes and be complementary to the localization techniques used within the sensing cloud relying purely on round trip delays. 


\subsection{Use of commercial WSN for space exploration}

To our best knowledge, to date, commercially available WSN are capable of [11]:

- Ad/hoc functionalities;

- self-organization and reconfiguration;

- less than $1 \mathrm{~km}$ range communication.

Therefore there are three essential needs of WSN for space exploration that are not yet met by commercial WSN:

- communication at distances up to $10 \mathrm{~km}$;

- localization functionalities (preferably without relying on GPS/Galileo-type technologies or on RSS);

- dynamic rapid self-organization.

Commercial WSN products can be used now to demonstrate on Earth most aspects of an exploration mission. They are not yet mature enough to be used in space to collect reliable scientific data. To develop WSN for space exploration an effort would have to be made towards: increasing the communication range and implementing localization functionalities, and understanding radiation and environmental constraints and associated packaging and shielding issues.

\section{Conclusion}

WSN is a new technology for space exploration that has yet to prove the numerous advantages one can expect: low cost, accurate measurements over a large surface or volume, short set-up time of a mission, high reliability through redundancy.

WSN will have to be optimized to meet the specific requirements of space exploration: need for selflocalization and reliable long distance communication (few km).

Localization techniques are dependant on the mission scenario. A LIDAR based technique can be well suited to locate nodes on the ground of a planet, while RF based techniques better suited for a cloud of moving nodes. As RF based techniques require several reference nodes, the reference nodes can be located from the spacecraft by a technique combining distance and angular measurement such as LIDAR or RF [9].

The numerical performance simulations demonstrate the feasibility to use LIDAR (laser radar) for locating nodes. Even for the case of Mars, with its attenuating atmosphere, such location requires LIDAR specifications compatible with the present commercial technology for compact lasers and detectors. The studied location technique does not necessary require complication of the nodes.

RF based localization techniques are easier to implement and more accurate when there is no multipath such as in free space or in atmosphere. For a cloud of moving nodes, round trip delay measurements from each node is a promising approach that can provide accurate positioning using commercial quartz time reference. Implementing RF based localization in a WSN for space exploration does not require major node computational complication since the localization algorithm can be centralized in the base node or in the spacecraft.

Each mission will require highly optimized nodes, with sensors, communications, and packaging suited to the environment the WSN will be operating in, and to the characteristics that must be measured. For each mission, the data acquisition and transmission of the WSN will have to be tested on Earth in an environment as similar as possible to the one that would encounter the nodes when deployed in space.

\section{Acknowledgments}

The authors wish to acknowledge financial support from the Sixth European Program for the e-CUBES project.

\section{References}

[1] R. Magness, Wireless onboard spacecraft and in space exploration, TEC-E wireless technology dossier, ESA-ESTEC, ref.TOSE-1B-DOS-4, 2006.

[2] European project e-CUBES 〈http://ecubes.epfl.ch/public/〉.

[3] T.S. Rappaport, Wireless Communications-Principles and Practice, second ed., Prentice-Hall, New York, 2002.

[4] W. Ye, J. Heidemann, D. Estrin, An energy-efficient MAC protocol for wireless sensor networks, Presented at Proceedings-IEEE INFOCOM, New York, NY, 2002.

[5] A. Sinha, A. Chandrakasan, Dynamic power management in wireless sensor networks, IEEE Design and Test of Computers 18 (2001) 62-74.

[6] B.H. Calhoun, D.C. Daly, N. Verma, D.F. Finchelstein, D.D. Wentzloff, A. Wang, S.-H. Cho, A.P. Chandrakasan, Design considerations for ultra-low energy wireless microsensor nodes, IEEE Transactions on Computers 54 (2005) 727-740.

[7] W. Stallings, Wireless Communications and Networks, second ed., Pearson Prentice-Hall, 2005.

[8] A. Rogoyski, B. Skidmore, V. Maheswaran, I. Wright, J. Zarnecki, C. Pillinger, Wide area microprobe analyser (WAMPA), Acta Astronautica 59 (2006) 1029-1038.

[9] A. Schiele, J. Laycock, A.W. Ballard, M. Cosby, E. Picardi, DALOMIS: A Data Transmission And Localisation System For Microprobes Swarms, European Space Agency, (Special Publication) ESA SP 2005, pp. 693-703. 
[10] N. Patwari, J.N. Ash, S. Kyperountas, A.O. Hero, R.L. Moses, N.S. Correal, Locating the nodes: cooperative localization in wireless sensor networks, IEEE Signal Processing Magazine 22 (4) (2005) 54-69.

[11] T. Vladimirova, C.P. Bridges, G. Prassinos, X. Wu, K. Sidibeh, D.J. Barnhart, A.-H. Jallad, J.R. Paul, V. Lappas, A. Baker, K. Maynard, R. Magness, Characterising wireless sensor motes for space applications, Presented at Second NASA/ESA Conference on Adaptive Hardware and Systems, IEEE Computer Society, Edinburgh, August 2007.

[12] D. Niculescu, Positioning in ad hoc sensor networks, IEEE Network 18 (4) (2004) 24-29.

[13] G. Sun, J. Chen, W. Guo, K.J.R. Liu, Signal processing techniques in network-aided positioning, IEEE Signal Processing Magazine 22 (4) (2005) 12-23.

[14] R.J. Fontana, Recent system applications of short-pulse ultrawideband (UWB) technology, IEEE Transactions on Microwave Theory and Techniques 52 (2004) 2087-2104.

[15] S. Dubowsky, K. Iagnemma, S. Liberatore, D. Lambeth, J.S. Plante, P. Boston, A concept mission: microrobots for large-scale planetary surface and subsurface exploration, in: Proceedings of the 2005 Space Technology and Applications International Forum (STAIF), Albuquerque, NM, February 2005.

[16] T. Yoshimitsu, Development of autonomous rover for asteroid surface exploration, Presented at Proceedings-IEEE
International Conference on Robotics and Automation, New Orleans, LA, 2004.

[17] S. Eckerley, J. Schalk, O. Coumar, K. Haria, The EADS micropack, 5th round table on micro/nano technologies for space, ESA-ESTEC, 2005.

[18] F. Luca, Environmental monitoring by laser radar, in: S.B. Larkin (Ed.), Lasers and Electro-Optics Research at the Cutting Edge, Nova Publishers, New York, 2007, pp. 119-171.

[19] J.B. Pollack, Properties of dust in the Martian atmosphere and its effect on temperature structure, in: A. Kliore (Ed.), The Mars Reference Atmosphere, Published for the Committee of Space Research by Pergamon Press, 1982, pp. 45-56, (Chapter III).

[20] J.B. Pollack, E.M. Ockert-Bell, M.K. Schepard, Viking Lander image analysis of Martian atmospheric dust, Journal of Geophysics 100 (E3) (1995) 5235-5250.

[21] C. Niclass, A. Rosckas, P.A. Besse, E. Charbon, Toward a 3D camera based on single photon avalanche diodes, IEEE Journal of selected topics in Quantum Electronics 10 (4) (2004) 796802.

[22] S.M. Kay, Fundamentals of Statistical Signal Processing: Estimation Theory, Prentice-Hall Signal Processing Series, Upper Saddle River, New Jersey, 1993.

[23] C. Botteron, A. Høst-Madsen, M. Fattouche, Cramer-Rao bounds for the estimation of multipath parameters and mobiles' positions in asynchronous DS-CDMA systems, IEEE Transactions on Signal Processing 52 (4) (2004) 862-875. 